\title{
PERSPECTIVE OF COASTAL VEGETATION PATCHES WITH TOPOGRAPHY VARIATIONS FOR TSUNAMI PROTECTION IN 2D - NUMERICAL MODELING
}

\author{
N. A. K. NANDASENA ${ }^{1)}$, Norio TANAKA ${ }^{2)}$ and Katsutoshi TANIMOTO ${ }^{3)}$ \\ ${ }^{1}$ Member of JSCE, B.Sc.Eng (Hons), Graduate School of Science and Engineering, Saitama University (255 Shimo-okubo, \\ Sakura-ku, Saitama 338-8570, Japan) \\ ${ }^{2}$ Member of JSCE, Dr. of Eng., Professor, Graduate School of Science and Engineering, Saitama University (255 Shimo- \\ okubo, Sakura-ku, Saitama 338-8570, Japan) \\ ${ }^{3}$ Member of JSCE, Dr. of Eng., Professor, Graduate School of Science and Engineering, Saitama University (255 Shimo- \\ okubo, Sakura-ku, Saitama 338-8570, Japan)
}

\begin{abstract}
Effectiveness of two types of densely grown coastal vegetation, namely Pandanus odoratissimus and Casuarina equisetifolia were investigated with different ground slopes, against a tsunami event in 2-D numerical simulation. Dimensions of vegetation patches and gap between them were changed rationally in order to represent possible vegetation shapes available on hinterland. Both vegetations showed similar trend of hydraulic properties variations. It was observed that reduction of maximum current velocity is higher than the reduction of maximum water depth behind vegetation patch. Horizontal run-up length is reduced with the patch size increases for very mild ground slope, but when slope becomes steep, gravity effect is dominant than vegetation effect on run-up length. Relatively big patches with narrow gap are identified as destructive forms. In fact maximum current velocity is increased at gap-outlet, gap-inlet and middle of the gap when tsunami flows landward, towards offshore and in both directions respectively compared to the bare land.
\end{abstract}

Key words : vegetation patch, tsunami, topography effect, 2D - numerical modeling, bare land

\section{INTRODUCTION}

Coastal vegetation is being introduced as a softcountermeasure against the tsunami impact. This technique can be promisingly applied to developing countries as it involves initial less capital investment in comparison with other sophisticated coastal protection measures like artificial structures. Researchers identified coastal vegetation can retardate considerable amount of violent energy carried with gigantic tsunami waves in historic tsunami field surveys. In fact, not all coastal vegetation species, but densely grown vegetation types, like Pandanus odoratissimus, Casuarina equisetifolia and types of Mangrove species where behind of those vegetation, damaged considerably reduced, Tanaka et al. ${ }^{3)}$, Mascarenhas and Jayakumar". Nandasena et al. ${ }^{2)}$ conducted 1 - D numerical simulation on selected coastal species and it was identified that densely grown coastal vegetation species can reduce current velocity than water depth remarkably behind those vegetation compared to sparsely grown coastal vegetations (or plantations). Tanaka et al. ${ }^{4)}$ also investigated hydraulic properties through coastal vegetation and their breaking conditions in a tsunami event, explored that Casuarina equisetifolia and Pandanus odoratissimus exhibited a strong potential to resist tsunami force in a two-layer arrangement. Above all calculations were confined to 1 - D numerical simulations with the assumption of infinitely long vegetation belt in the transverse direction. This paper address the effectiveness of coastal vegetation patches and gap (between vegetation patches) effect more comprehensively in 2 - D numerical simulation to bridge the gap between 1 - D and real-ground situation.

\section{MATERIALS AND METHODS}

\section{(1) Governing equations}

To describe the flow through the vegetation, twodimensional depth integrated Continuity and Momentum equations in two perpendicular directions are used. Nandasena et al. ${ }^{1)}$ improved governing equations with the full effect of porosity due to solidity of vegetation. Here the governing equations (1) continuity (2) and (3) momentum equations in $x$ 
and $y$ directions respectively, are replaced by discharge and water depth terms in order to facilitate the simulation codes.

$$
\begin{gathered}
\theta_{h} \frac{\partial h}{\partial t}+\frac{\partial Q_{x}}{\partial x}+\frac{\partial Q_{y}}{\partial y}=0 \\
\theta_{h} \frac{\partial Q_{x}}{\partial t}+\frac{\partial\left(\frac{Q_{x}^{2}}{h}\right)}{\partial x}+\frac{\partial\left(\frac{Q_{x} Q_{y}}{h}\right)}{\partial y}+\theta_{h} g h \frac{\partial h}{\partial x} \\
+\theta_{h}^{3 / 2} g h \frac{\partial z}{\partial x}+\theta_{b} \sqrt{\theta_{h}} \frac{\tau_{x}}{\rho}+\frac{\theta_{h}^{3 / 2}}{\rho} \sum_{i=1}^{k} f_{x i}=0 \\
\theta_{h} \frac{\partial Q_{y}}{\partial t}+\frac{\partial\left(\frac{Q_{x} Q_{y}}{h}\right)}{\partial x}+\frac{\partial\left(\frac{Q_{y}^{2}}{h}\right)}{\partial y}+\theta_{h} g h \frac{\partial h}{\partial y} \\
+\theta_{h}^{3 / 2} g h \frac{\partial z}{\partial y}+\theta_{b} \sqrt{\theta_{h}} \frac{\tau_{y}}{\rho}+\frac{\theta_{h}^{3 / 2}}{\rho} \sum_{i=1}^{k} f_{y i}=0
\end{gathered}
$$

Where, $Q_{x}=$ discharge in $x$ direction, $Q_{y}=$ discharge in $y$ direction, $Q=\sqrt{Q_{x}^{2}+Q_{y}^{2}}, h=$ water depth, $z=$ bed elevation measured from the selected datum, $\tau_{x}, \tau_{y}=$ bed resistance per unit area in $x$ and $y$ direction respectively, $\sum_{i=1}^{k} f_{x i}, \sum_{i=1}^{k} f_{y i}=$ resistance induced by vegetation per unit area (drag and inertia) in $x$ and $y$ direction respectively, $\rho=$ sea water density $(=1024$ $\left.\mathrm{kg} / \mathrm{m}^{3}\right), g=$ gravitational acceleration, $\theta_{h}, \theta_{b}=$ depth averaged and bed aerial porosities respectively.

$$
\begin{aligned}
& \theta_{h}=\left(1-\frac{\pi b_{h}^{2} m}{4}\right) \\
& \theta_{b}=\left(1-\frac{\pi b_{b}^{2} m}{4}\right)
\end{aligned}
$$

Where, $m=$ tree density (number of trees per unit area), $b_{h}=$ depth averaged tree diameter at water depth $h, b_{b}$ $=$ equivalent tree diameter on the ground. Bed resistance $(\tau)$, drag force $\left(F_{d}\right)$ and inertia force $\left(F_{i}\right)$ 's $x$ and $y$ components per unit area are reformulated to compatible with governing equations, respectively as follows.

$$
\begin{gathered}
\tau_{x}=\rho g n^{2} Q_{x} \sqrt{Q_{x}^{2}+Q_{y}^{2}} /\left(\theta_{h} h^{7 / 3}\right) \\
\tau_{y}=\rho g n^{2} Q_{y} \sqrt{Q_{x}^{2}+Q_{y}^{2}} /\left(\theta_{h} h^{7 / 3}\right) \\
F_{d x}=m \frac{1}{2} \rho C_{d-a l l} b_{r e f} h Q_{x} \sqrt{Q_{x}^{2}+Q_{y}^{2}} / h^{2} \theta_{h} \\
F_{d y}=m \frac{1}{2} \rho C_{d-a l l} b_{r e f} h Q_{y} \sqrt{Q_{x}^{2}+Q_{y}^{2}} / h^{2} \theta_{h} \\
F_{i x}=m \rho C_{M} \forall \frac{d\left(Q / h \sqrt{\theta_{h}}\right)}{d t} \frac{Q_{x}}{\sqrt{Q_{x}^{2}+Q_{y}^{2}}}
\end{gathered}
$$

$$
F_{i y}=m \rho C_{M} \forall \frac{d\left(Q / h \sqrt{\theta_{h}}\right)}{d t} \frac{Q_{y}}{\sqrt{Q_{x}^{2}+Q_{y}^{2}}}
$$

Where, $C_{d \text {-all }}$ (for more details refer, Tanaka et al. ${ }^{3)}$ ) is defined as follows.

$$
C_{d-a l l}=C_{d-r e f} \frac{1}{h} \int_{0}^{h} \frac{b(y)}{b_{r e f}} \frac{C_{d}(y)}{C_{d-r e f}} d y
$$

Where, $b(y)=$ tree diameter at height, $y, C_{d}(y)=$ drag coefficient at height, $y, b_{\text {ref }}=$ reference tree diameter at height, $1.2 \mathrm{~m}, C_{d-r e f}=$ reference drag coefficient $(=1.0)$ $C_{M}=$ reference inertia coefficient $(=2.0), n=$ Manning's roughness coefficient $(=0.025), \quad \forall=$ submerged volume of a tree due to water depth.

\section{(2) Selection of parameters}

\subsection{Vegetation species}

Following the outcomes of Tanaka et al. ${ }^{3), 4)}$ and Nandasena et al. ${ }^{2)}$, most suitable coastal vegetation species to mitigate the tsunami impact, Pandanus odoratissimus and Casuarina equisetifolia (young specie) were selected. Physical characteristics of Pandanus and Casuarina are, average tree height, $8 \mathrm{~m}$ and $10 \mathrm{~m}$, average tree density, 0.31 and 0.44 number of trees per unit area (in square arrangement) and average tree diameter at $1.2 \mathrm{~m}$ above ground level, $0.16 \mathrm{~m}$ and $0.15 \mathrm{~m}$, respectively.

\subsection{Topography, vegetation patches and tsunami condition}

Fig. 1 shows the cross section of bed profile along the tsunami direction, was selected for the simulation. Three different slopes, very mild (1:1000), typical (1:100) and relatively steep (1:50) extending from the shoreline, were chosen to identify the hydraulic properties changes on the topography variations. Fig. 2 shows the plan area of the vegetation patches arraignment in which line A-A indicates the vegetation front and B-B limits the rear boundary. Lateral boundaries and distance from them to near boundary of patch were fixed $(50 \mathrm{~m})$ as shown in Fig. 2 in which symmetry is established. It is assumed the distance is sufficient to minimize the disturbance to the results by lateral boundaries' reflection. Tsunami wave height $=$ $5 \mathrm{~m}$ and period $=20 \mathrm{~min}$ were assumed at $50 \mathrm{~m}$ water depth, respectively. Discretization length was $5 \mathrm{~m}$ for both $\mathrm{x}$ and $\mathrm{y}$ directions and simulation was restricted to a complete inundation on the ground. Water depth and current velocity ( $\mathrm{U}$ - tsunami direction, or X component, $\mathrm{V}$ - perpendicular to tsunami direction, or $\mathrm{Y}$ - component) were computed at five locations (1 


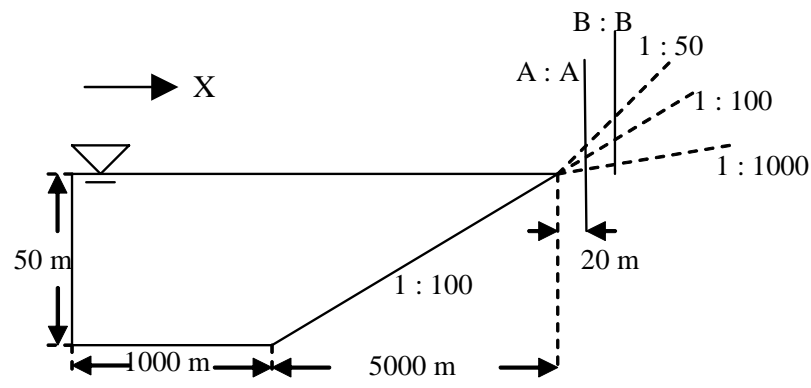

Fig. 1 Sketch of bed profile with the vegetation boundaries

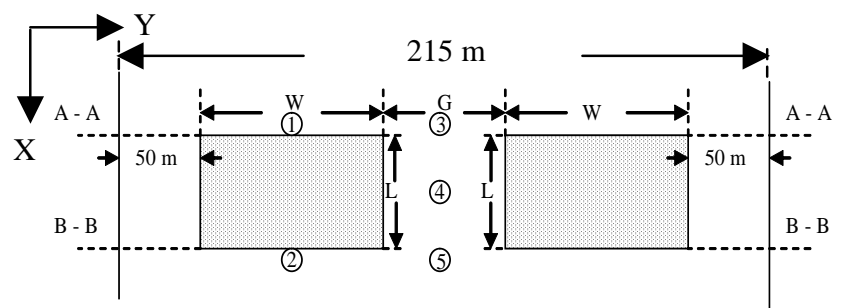

Fig. 2 Sketch of vegetation patches arrangement

to 5), of which locations 1 and 2 were selected to explore the effectiveness of the vegetation patch while the locations 3,4 and 5 were employed to check vegetation gap effect on the tsunami flow. W (width of the patch normal to the tsunami direction), $\mathbf{L}$ (length of the patch along tsunami direction) and $\mathbf{G}$ (gap) between them were altered as follows in Table 1.

Table 1 Vegetation patch dimension

\begin{tabular}{|c|c|c|c|c|}
\hline & L = 50 $\mathbf{~}$ & $\mathbf{W}=\mathbf{2 5} \mathbf{~ m}$ & $\mathbf{G}=\mathbf{6 5} \mathbf{~ m}$ & Remarks \\
\hline Case 1 & L & W & G & $\begin{array}{c}\text { Wide gap - } \\
\text { small patches }\end{array}$ \\
\hline Case 2 & L & 2W & $3 \mathrm{G} / 13$ & $\begin{array}{c}\text { Narrow gap - } \\
\text { small patches }\end{array}$ \\
\hline Case 3 & 2L & W & G & $\begin{array}{c}\text { Wide gap - big } \\
\text { patches }\end{array}$ \\
\hline Case 4 & 2L & 2W & $3 \mathrm{G} / 13$ & $\begin{array}{c}\text { Narrow gap - } \\
\text { big patches }\end{array}$ \\
\hline
\end{tabular}

\section{RESULTS AND DISCUSSION}

Pandanus odoratissimus can be broken or bent, when tsunami water depth is higher than $80 \%$ of tree height and Casuarina equisetifolia is stronger than Pandanus, Tanaka et al. ${ }^{4}$. Hence, it was assumed that the vegetation was hardly any broken in this study. Inertia and drag coefficients were constant and dynamic behavior (bending and shaking of trees) of the vegetation during a tsunami event was not considered. Table 2, 4 and 6 show the maximum water depth variation for each case compared to bare land (without vegetation). For each case, maximum water depth for bare land and then the ratio of maximum water depth with vegetation to maximum water depth for bare land, i.e. (Vegetation/Bare land) are tabulated. Table 3, 5 and 6 show maximum current velocity variation, (+ve) means landward, (-ve) indicates the offshore direction respectively. For each case, maximum current velocity for bare land (two maximums, one per landward and other per offshore direction) and ratio of maximum current velocity with vegetation to maximum current velocity for bare land (Vegetation/Bare land); first ratio, landward and the second, offshore direction represented, respectively. Table 2 and 3 shows the maximum water depth and maximum current velocity variation for the $1: 1000$ ground slope respectively. Note that only location 3 is

Table 2 Maximum water depth variation for $1: 1000$ slope, $\mathrm{P}=$ Pandanus, $\mathrm{C}=$ Casuarina, locations and cases are as shown in Fig. 2 and Table 1 respectively.

\begin{tabular}{|c|c|c|c|c|c|c|c|c|c|c|}
\hline Location & \multicolumn{2}{|c|}{1} & \multicolumn{2}{|c|}{2} & \multicolumn{2}{|c|}{3} & \multicolumn{2}{|c|}{4} & \multicolumn{2}{|c|}{5} \\
\hline Case 1 & $\mathbf{P}$ & $\mathrm{C}$ & $\mathbf{P}$ & C & $\mathbf{P}$ & $\mathbf{C}$ & $\mathbf{P}$ & $\mathrm{C}$ & $\mathbf{P}$ & $\mathrm{C}$ \\
\hline $\begin{array}{c}\text { Bare land } \\
(\mathrm{m})\end{array}$ & \multicolumn{2}{|c|}{4.23} & \multicolumn{2}{|c|}{4.16} & \multicolumn{2}{|c|}{4.23} & \multicolumn{2}{|c|}{4.2} & \multicolumn{2}{|c|}{4.16} \\
\hline $\begin{array}{l}\text { (Vegetation / } \\
\text { Bare land) }\end{array}$ & 1.12 & 1.09 & 0.91 & 0.94 & 1.07 & 1.05 & 1.00 & 1.01 & 0.93 & 0.96 \\
\hline \multicolumn{11}{|l|}{\begin{tabular}{|l|} 
Case 2 \\
\end{tabular}} \\
\hline $\begin{array}{c}\text { Bare land } \\
\text { (m) }\end{array}$ & \multicolumn{2}{|c|}{4.23} & \multicolumn{2}{|c|}{4.16} & \multicolumn{2}{|c|}{4.23} & \multicolumn{2}{|c|}{4.2} & \multicolumn{2}{|c|}{4.16} \\
\hline $\begin{array}{l}\text { (Vegetation / } \\
\text { Bare land) }\end{array}$ & 1.2 & 1.15 & 0.86 & 0.90 & 1.19 & 1.14 & 1.04 & 1.04 & 0.86 & 0.91 \\
\hline \multicolumn{11}{|l|}{ Case 3} \\
\hline $\begin{array}{c}\text { Bare land } \\
\text { (m) }\end{array}$ & \multicolumn{2}{|c|}{4.23} & \multicolumn{2}{|c|}{4.09} & \multicolumn{2}{|c|}{4.23} & \multicolumn{2}{|c|}{4.16} & \multicolumn{2}{|c|}{4.09} \\
\hline $\begin{array}{l}\text { (Vegetation / } \\
\text { Bare land) }\end{array}$ & 1.14 & 1.11 & 0.89 & 0.92 & 1.10 & 1.08 & 0.96 & 0.99 & 0.90 & 0.92 \\
\hline \multicolumn{11}{|l|}{ Case 4} \\
\hline \begin{tabular}{|c|} 
Bare land \\
(m)
\end{tabular} & \multicolumn{2}{|c|}{4.23} & \multicolumn{2}{|c|}{4.09} & \multicolumn{2}{|c|}{4.23} & \multicolumn{2}{|c|}{4.16} & \multicolumn{2}{|c|}{4.09} \\
\hline $\begin{array}{l}\text { (Vegetation / } \\
\text { Bare land) }\end{array}$ & 1.24 & 1.19 & 0.81 & 0.87 & 1.23 & 1.19 & 1.02 & 1.03 & 0.82 & 0.87 \\
\hline
\end{tabular}

Table 3 Maximum current velocity (U) variation for $1: 1000$ slope, $\mathrm{P}=$ Pandanus, $\mathrm{C}=$ Casuarina , locations and cases are as shown in Fig. 2 and Table 1 respectively. (-) indicates the offshore currents velocity.

\begin{tabular}{|c|c|c|c|c|c|c|c|c|c|c|}
\hline \multirow{2}{*}{\begin{tabular}{|c|} 
Location \\
Case 1 \\
\end{tabular}} & \multicolumn{2}{|c|}{1} & \multicolumn{2}{|c|}{2} & \multicolumn{2}{|c|}{3} & \multicolumn{2}{|c|}{4} & \multicolumn{2}{|c|}{5} \\
\hline & $\mathbf{P}$ & $\mathrm{C}$ & $\mathbf{P}$ & $\mathrm{C}$ & $\mathbf{P}$ & $\mathrm{C}$ & $\mathbf{P}$ & $\mathrm{C}$ & $\mathbf{P}$ & $\mathrm{C}$ \\
\hline $\begin{array}{c}\text { Bare land } \\
(\mathbf{m} / \mathbf{s})\end{array}$ & \multicolumn{2}{|c|}{$4.48,-2.9$} & \multicolumn{2}{|c|}{$4.53,-2.42$} & \multicolumn{2}{|c|}{$4.48,-2.9$} & \multicolumn{2}{|c|}{$4.52,-2.61$} & \multicolumn{2}{|c|}{$4.53,-2.42$} \\
\hline \begin{tabular}{|c}
$($ Vegetation / \\
Bare land)
\end{tabular} & $\begin{array}{l}0.89, \\
0.39 \\
\end{array}$ & $\begin{array}{l}0.96, \\
0.63 \\
\end{array}$ & $\begin{array}{l}0.38, \\
0.66 \\
\end{array}$ & \begin{tabular}{|l}
0.58, \\
0.80 \\
\end{tabular} & $\begin{array}{l}0.98, \\
1.12 \\
\end{array}$ & $\begin{array}{l}0.98, \\
1.06\end{array}$ & $\begin{array}{l}0.99, \\
1.05 \\
\end{array}$ & $\begin{array}{l}0.97, \\
1.00\end{array}$ & $\begin{array}{l}1.19, \\
0.85 \\
\end{array}$ & $\begin{array}{l}1.12, \\
0.88 \\
\end{array}$ \\
\hline \multicolumn{11}{|l|}{ Case 2} \\
\hline $\begin{array}{c}\text { Bare land } \\
(\mathrm{m} / \mathrm{s})\end{array}$ & \multicolumn{2}{|c|}{$4.48,-2.9$} & \multicolumn{2}{|c|}{$4.53,-2.42$} & \multicolumn{2}{|c|}{$4.48,-2.9$} & \multicolumn{2}{|c|}{$4.52,-2.61$} & \multicolumn{2}{|c|}{$4.53,-2.42$} \\
\hline $\begin{array}{c}\text { (Vegetation / } \\
\text { Bare land) }\end{array}$ & $\begin{array}{l}0.89, \\
0.52 \\
\end{array}$ & $\begin{array}{l}0.96 \\
0.73\end{array}$ & $\begin{array}{l}0.53, \\
0.48\end{array}$ & $\begin{array}{l}0.68, \\
0.67\end{array}$ & $\begin{array}{l}0.98, \\
1.29\end{array}$ & $\begin{array}{l}0.98, \\
1.18\end{array}$ & $\begin{array}{l}1.06 \\
1.04\end{array}$ & $\begin{array}{l}1.00, \\
1.00\end{array}$ & $\begin{array}{l}1.32, \\
0.58\end{array}$ & $\begin{array}{l}1.22, \\
0.69\end{array}$ \\
\hline \multicolumn{11}{|l|}{ Case 3} \\
\hline $\begin{array}{c}\text { Bare land } \\
(\mathbf{m} / \mathbf{s})\end{array}$ & \multicolumn{2}{|c|}{$4.48,-2.9$} & \multicolumn{2}{|c|}{$4.54,-2.23$} & \multicolumn{2}{|c|}{$4.48,-2.9$} & \multicolumn{2}{|c|}{$4.53,-2.45$} & \multicolumn{2}{|c|}{$4.54,-2.23$} \\
\hline $\begin{array}{c}(\text { Vegetation / } \\
\text { Bare land) }\end{array}$ & $\begin{array}{l}0.89, \\
0.38 \\
\end{array}$ & $\begin{array}{l}0.96, \\
0.57\end{array}$ & $\begin{array}{l}0.31, \\
0.66\end{array}$ & $\begin{array}{l}0.50, \\
0.80\end{array}$ & $\begin{array}{l}0.98, \\
1.10\end{array}$ & $\begin{array}{c}0.98, \\
1.07\end{array}$ & $\begin{array}{l}1.07, \\
1.12\end{array}$ & $\begin{array}{l}1.03, \\
1.05\end{array}$ & $\begin{array}{l}1.25, \\
0.83\end{array}$ & $\begin{array}{l}1.19, \\
0.85\end{array}$ \\
\hline \multicolumn{11}{|l|}{ Case 4} \\
\hline $\begin{array}{l}\text { Bare land } \\
(\mathrm{m} / \mathrm{s})\end{array}$ & \multicolumn{2}{|c|}{$4.48,-2.9$} & \multicolumn{2}{|c|}{$4.54,-2.23$} & \multicolumn{2}{|c|}{$4.48,-2.9$} & \multicolumn{2}{|c|}{$4.53,-2.45$} & \multicolumn{2}{|c|}{$4.54,-2.23$} \\
\hline \begin{tabular}{|c} 
(Vegetation / \\
Bare land)
\end{tabular} & $\begin{array}{l}0.89, \\
0.44 \\
\end{array}$ & $\begin{array}{l}0.96, \\
0.64\end{array}$ & $\begin{array}{l}0.40, \\
0.48\end{array}$ & $\begin{array}{l}0.55, \\
0.65\end{array}$ & $\begin{array}{l}0.98, \\
1.28\end{array}$ & $\begin{array}{c}0.98, \\
1.20\end{array}$ & $\begin{array}{l}1.23, \\
1.09\end{array}$ & $\begin{array}{l}1.13, \\
1.03\end{array}$ & $\begin{array}{l}1.42, \\
0.50\end{array}$ & $\begin{array}{l}1.32, \\
0.64\end{array}$ \\
\hline
\end{tabular}


the same in each case, but locations $1,2,4$, and 5 are different even in the bare land case because of the different patch size in corresponding comparison case. It was observed that relatively big vegetation patches with narrow gap (case 4) reflected high water depth at the vegetation front compared with other cases for very mild slope condition. Though Casuarina has high tree density than Pandanus, maximum water depth recorded at vegetation front belonged to later, as its total projection area was high and thus blockage was high. There was no significant difference of maximum water depth variation through vegetation and along the gap for small patches with wide gap. However, when the patch size increased and gap became narrow, reduction of maximum water depths behind the vegetation and gap-outlet (loc 5) were remarkable as

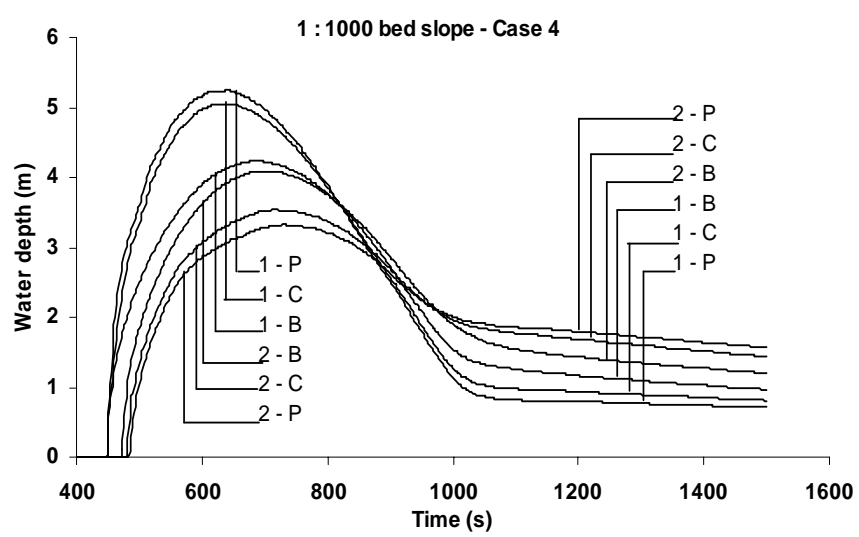

Fig. 3 Temporal water depth variation for Case 4 of 1 : 1000 slope, $\mathrm{P}=$ Pandanus, $\mathrm{C}=$ Casuarina, $\mathrm{B}=$ Bare land and numbers represent locations as indicated in Fig. 2

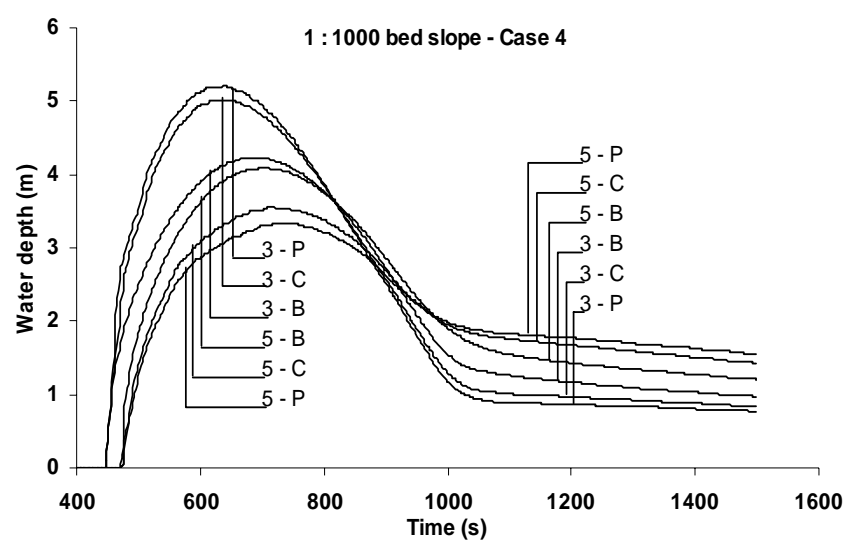

Fig. 4 Temporal water depth variation for Case 4 of $1: 1000$ slope, $\mathrm{P}=$ Pandanus, $\mathrm{C}=$ Casuarina, $\mathrm{B}=$ Bare land and numbers represent locations as indicated in Fig. 2

shown in Table 2. Maximum current velocity toward land was increased at gap-outlet (loc 5) for inflow and the maximum current velocity offshore direction was magnified at gap - inlet (loc 3) for reversed flow in comparison with bare land. This phenomenon was dominant in big patches with narrow gap. Contrary to small patches with gaps, big patches with gaps showed maximum current velocity increment in the mid of the gap for both inflow and out flow compared to bare land. Fig. 3 and 4 show the temporal water depth variation at front of (loc 1) and behind (loc 2) the vegetation and through the gap (i.e. gap - inlet (loc 3), mid of the gap (loc 4) and gap - outlet (loc 5)) respectively. It was noticed that very mild slope can delay the seawater retrieving to the offshore and it would remain until next tsunami wave comes. This is due to vegetation resistance and also effect of feeble gravitational support to accelerate the backflow. Fig. 5 and 6 illustrate corresponding current velocity variation at vegetation area and through the gap. It was dominant that sudden dropped down of current velocity profile when the wave collided on vegetation

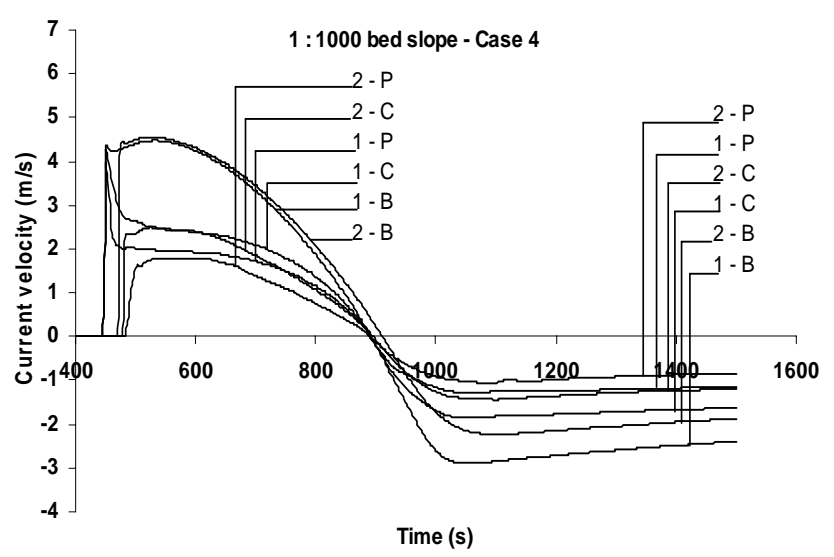

Fig. 5 Temporal current velocity (U) variation for Case 4 of 1 : 1000 slope, $\mathrm{P}=$ Pandanus, $\mathrm{C}=$ Casuarina, $\mathrm{B}=$ Bare land and numbers represent locations as indicated in Fig. 2

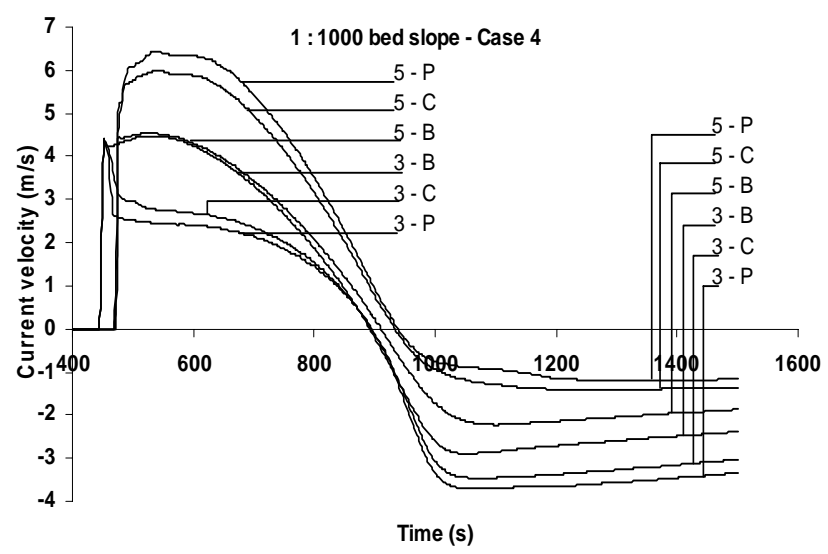

Fig. 6 Temporal current velocity (U) variation for Case 4 of 1 : 1000 slope, $\mathrm{P}=$ Pandanus, $\mathrm{C}=$ Casuarina, $\mathrm{B}=$ Bare land and numbers represent locations as indicated in Fig. 2 
front as shown in Fig. $5(1-\mathrm{P}$ and $1-\mathrm{C})$ while this effect transferred to gap - inlet where current velocity fell down at the begin as shown in Fig. 6 (3 - P and 3 C). It was noticed that current velocity at the gapoutlet (loc 5) increased than bare land at flow in landward and similar phenomenon occurred at gapinlet (loc 3) when flow reversed. These results reveal that big vegetation patches with narrow gap exacerbate the damage situation through and the vicinity of gap area as current velocity increment causes to magnify the damage force on the structures and properties. Table 4 and 5 shows the maximum water depth variation and maximum current velocity variation for 1 : 100 ground slope with the different vegetation

Table 4 Maximum water depth variation for $1: 100$ slope, $\mathrm{P}=$ Pandanus, $\mathrm{C}=$ Casuarina, locations and cases are as shown in Fig. 2 and Table 1 respectively.

\begin{tabular}{|c|c|c|c|c|c|c|c|c|c|c|}
\hline Location & \multicolumn{2}{|c|}{1} & \multicolumn{2}{|c|}{2} & \multicolumn{2}{|c|}{3} & \multicolumn{2}{|c|}{4} & \multicolumn{2}{|c|}{5} \\
\hline Case 1 & $\mathbf{P}$ & $\mathrm{C}$ & $\mathbf{P}$ & $\mathrm{C}$ & $\mathbf{P}$ & $\mathrm{C}$ & $\mathbf{P}$ & $\mathrm{C}$ & $\mathbf{P}$ & $\mathbf{C}$ \\
\hline $\begin{array}{l}\text { Bare land } \\
\text { (m) }\end{array}$ & \multicolumn{2}{|c|}{6.04} & \multicolumn{2}{|c|}{5.59} & \multicolumn{2}{|c|}{6.04} & \multicolumn{2}{|c|}{5.84} & \multicolumn{2}{|c|}{5.59} \\
\hline \begin{tabular}{|c|} 
Vegetation / \\
Bare land) \\
\end{tabular} & 0.99 & 0.99 & 0.98 & 0.99 & 0.99 & 0.99 & 0.99 & 0.99 & 0.98 & 0.99 \\
\hline \multicolumn{11}{|l|}{\begin{tabular}{|l|} 
Case 2 \\
\end{tabular}} \\
\hline $\begin{array}{l}\text { Bare land } \\
\text { (m) }\end{array}$ & \multicolumn{2}{|c|}{6.04} & \multicolumn{2}{|c|}{5.59} & \multicolumn{2}{|c|}{6.04} & \multicolumn{2}{|c|}{5.84} & \multicolumn{2}{|c|}{5.59} \\
\hline \begin{tabular}{|c|} 
Vegetation / \\
Bare land) \\
\end{tabular} & 0.98 & 0.98 & 0.97 & 0.98 & 0.98 & 0.98 & 0.97 & 0.98 & 0.97 & 0.98 \\
\hline \multicolumn{11}{|l|}{ Case 3} \\
\hline $\begin{array}{l}\text { Bare land } \\
\text { (m) }\end{array}$ & \multicolumn{2}{|c|}{6.04} & \multicolumn{2}{|c|}{5.18} & \multicolumn{2}{|c|}{6.04} & \multicolumn{2}{|c|}{5.63} & \multicolumn{2}{|c|}{5.18} \\
\hline \begin{tabular}{|c|} 
(Vegetation / \\
Bare land) \\
\end{tabular} & 0.99 & 0.99 & 0.98 & 0.98 & 0.99 & 0.99 & 0.98 & 0.99 & 0.98 & 0.98 \\
\hline \multicolumn{11}{|l|}{ Case 4} \\
\hline $\begin{array}{c}\text { Bare land } \\
(\mathbf{m})\end{array}$ & \multicolumn{2}{|c|}{6.04} & \multicolumn{2}{|c|}{5.18} & \multicolumn{2}{|c|}{6.04} & \multicolumn{2}{|c|}{5.63} & \multicolumn{2}{|c|}{5.18} \\
\hline $\begin{array}{c}\text { (Vegetation / } \\
\text { Bare land) }\end{array}$ & 0.97 & 0.98 & 0.95 & 0.97 & 0.97 & 0.98 & 0.96 & 0.97 & 0.95 & 0.97 \\
\hline
\end{tabular}

Table 5 Maximum current velocity (U) variation for $1: 100$ slope, $\mathrm{P}=$ Pandanus, $\mathrm{C}=$ Casuarina, locations and cases are as shown in Fig. 2 and Table 1 respectively. (-) indicates the offshore currents velocity.

\begin{tabular}{|c|c|c|c|c|c|c|c|c|c|c|}
\hline \multirow{2}{*}{\begin{tabular}{|c|} 
Location \\
Case 1 \\
\end{tabular}} & \multicolumn{2}{|c|}{1} & \multicolumn{2}{|c|}{2} & \multicolumn{2}{|c|}{ 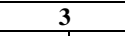 } & \multicolumn{2}{|c|}{7} & \\
\hline & $\mathbf{P}$ & C & $\mathbf{P}$ & C & $\mathbf{P}$ & $\mathbf{C}$ & $\mathbf{P}$ & C & $\mathbf{P}$ & C \\
\hline $\begin{array}{c}\text { Bare land } \\
(\mathrm{m} / \mathrm{s})\end{array}$ & \multicolumn{2}{|c|}{$4.22,-3.27$} & \multicolumn{2}{|c|}{$3.94,-3.16$} & \multicolumn{2}{|c|}{$4.22,-3.27$} & \multicolumn{2}{|c|}{$4.06,-3.22$} & \multicolumn{2}{|c|}{$3.94,-3.16$} \\
\hline & & \begin{tabular}{|l}
0.98, \\
0.59 \\
\end{tabular} & 31, & & & & \begin{tabular}{|l|}
1.00, \\
1.15 \\
\end{tabular} & & 1.05, & \\
\hline \multicolumn{11}{|l|}{ Case } \\
\hline $\begin{array}{r}\text { Bare l } \\
(\mathbf{m} /\end{array}$ & \multicolumn{2}{|c|}{$4.22,-3.27$} & \multicolumn{2}{|c|}{$3.94,-3.16$} & \multicolumn{2}{|c|}{$4.22,-3.27$} & \multicolumn{2}{|c|}{$4.06,-3.22$} & \multicolumn{2}{|c|}{$3.94,-3.16$} \\
\hline & & $\begin{array}{l}0.98, \\
0.76 \\
\end{array}$ & & $\begin{array}{r}0.68, \\
0.72 \\
\end{array}$ & $\begin{array}{l}00, \\
.52 \\
2\end{array}$ & $\begin{array}{l}1.00 \\
1.35 \\
\end{array}$ & \begin{tabular}{|l|}
1.10, \\
1.16 \\
\end{tabular} & $\begin{array}{l}1.02, \\
1.10 \\
\end{array}$ & $\begin{array}{l}.29, \\
.67 \\
\end{array}$ & $\begin{array}{l}1.1 \\
0.7 \\
\end{array}$ \\
\hline & \multirow{2}{*}{\multicolumn{2}{|c|}{$4.22,-3.27$}} & & & & & & & & \\
\hline $\begin{array}{c}\text { Bare lanc } \\
(\mathbf{m} / \mathbf{s})\end{array}$ & & & \multicolumn{2}{|c|}{$3.77,-3.05$} & \multicolumn{2}{|c|}{$4.22,-3.27$} & \multicolumn{2}{|c|}{$3.95,-3.17$} & \multicolumn{2}{|c|}{$3.77,-3.05$} \\
\hline & & & & 8, & 36, & .00 & $\begin{array}{l}04, \\
25 \\
\end{array}$ & & $\begin{array}{l}1.08, \\
0.89\end{array}$ & 0. \\
\hline & & & & & & & & & & \\
\hline $\begin{array}{r}\text { Bare } \\
(\mathrm{m}\end{array}$ & $\mid 4.22$ & 3.2 & 3. & 3.05 & 4.2 & -3.27 & 3.95 & -3.17 & 3.7 & -3. \\
\hline & & $\begin{array}{l}0.5 \\
0 .\end{array}$ & & $\begin{array}{l}0.59, \\
0.71\end{array}$ & $\begin{array}{l}1.00, \\
163\end{array}$ & 1.00, & \begin{tabular}{|l|}
1.27, \\
1.25 \\
\end{tabular} & 1.16 , & $\begin{array}{l}1.39, \\
0.65\end{array}$ & \begin{tabular}{|l|}
1.2 \\
0.7 \\
\end{tabular} \\
\hline
\end{tabular}

patch arrangements respectively. Inundated maximum water depth of $1: 100$ slope were considerably higher than the 1 : 1000 slope condition for bare land case. Reduction of maximum water depths were insignificant for small vegetation patches with wide gap on typical slope $(1: 100)$. But reduction of current velocity behind the small vegetation patch was considerable. There was no any difference between calculated maximum water depth at both ends of vegetation and through the gap. However change of maximum current velocity shows similar trend as observed in Table 3. Table 6 and 7 shows the maximum water depth variation and maximum current velocity variation for $1: 50$, relatively steep ground

Table 6 Maximum water depth variation for $1: 50$ slope, $\mathrm{P}=$ Pandanus, $\mathrm{C}=$ Casuarina, locations and cases are as shown in Fig. 2 and Table 1 respectively.

\begin{tabular}{|c|c|c|c|c|c|c|c|c|c|c|}
\hline Location & \multicolumn{2}{|c|}{1} & \multicolumn{2}{|c|}{2} & \multicolumn{2}{|c|}{3} & \multicolumn{2}{|c|}{4} & \multicolumn{2}{|c|}{5} \\
\hline Case 1 & $\mathbf{P}$ & C & $\mathbf{P}$ & $\mathrm{C}$ & $\mathbf{P}$ & C & $\mathbf{P}$ & C & $\mathbf{P}$ & $\mathbf{C}$ \\
\hline $\begin{array}{l}\text { Bare land } \\
\text { (m) }\end{array}$ & \multicolumn{2}{|c|}{6.46} & \multicolumn{2}{|c|}{5.42} & \multicolumn{2}{|c|}{6.46} & \multicolumn{2}{|c|}{5.99} & \multicolumn{2}{|c|}{5.42} \\
\hline $\begin{array}{l}\text { (Vegetation / } \\
\text { Bare land) }\end{array}$ & 1.00 & 1.00 & 1.00 & 1.00 & 1.00 & 1.00 & 1.00 & 1.00 & 1.00 & 1.00 \\
\hline \multicolumn{11}{|l|}{ Case 2} \\
\hline $\begin{array}{c}\text { Bare land } \\
(\mathrm{m})\end{array}$ & \multicolumn{2}{|c|}{6.46} & \multicolumn{2}{|c|}{5.42} & \multicolumn{2}{|c|}{6.46} & \multicolumn{2}{|c|}{5.99} & \multicolumn{2}{|c|}{5.42} \\
\hline $\begin{array}{l}\text { (Vegetation / } \\
\text { Bare land) }\end{array}$ & 0.99 & 0.99 & 0.99 & 0.99 & 0.99 & 0.99 & 0.99 & 0.99 & 0.99 & 0.99 \\
\hline \multicolumn{11}{|l|}{ Case 3} \\
\hline $\begin{array}{c}\text { Bare land } \\
(\mathrm{m})\end{array}$ & \multicolumn{2}{|c|}{6.46} & \multicolumn{2}{|c|}{4.48} & \multicolumn{2}{|c|}{6.46} & \multicolumn{2}{|c|}{5.52} & \multicolumn{2}{|c|}{4.48} \\
\hline $\begin{array}{l}\text { (Vegetation / } \\
\text { Bare land) }\end{array}$ & 1.00 & 1.00 & 1.00 & 1.00 & 1.00 & 1.00 & 1.00 & 1.00 & 1.00 & 1.00 \\
\hline \multicolumn{11}{|l|}{ Case 4} \\
\hline $\begin{array}{l}\text { Bare land } \\
(\mathrm{m})\end{array}$ & \multicolumn{2}{|c|}{6.46} & \multicolumn{2}{|c|}{4.48} & \multicolumn{2}{|c|}{6.46} & \multicolumn{2}{|c|}{5.52} & \multicolumn{2}{|c|}{4.48} \\
\hline $\begin{array}{l}\text { (Vegetation / } \\
\text { Bare land) }\end{array}$ & 0.99 & 0.99 & 0.99 & 0.99 & 0.99 & 0.99 & 0.99 & 0.99 & 0.99 & 0.99 \\
\hline
\end{tabular}

Table 7 Maximum current velocity (U) variation for $1: 50$ slope, $\mathrm{P}=$ Pandanus, $\mathrm{C}=$ Casuarina, locations and cases are as shown in Fig. 2 and Table 1 respectively. (-) indicates the offshore currents velocity.

\begin{tabular}{|c|c|c|c|c|c|c|c|c|c|c|}
\hline Location & \multicolumn{2}{|c|}{1} & \multicolumn{2}{|c|}{2} & \multicolumn{2}{|c|}{3} & \multicolumn{2}{|c|}{4} & \multicolumn{2}{|c|}{5} \\
\hline Case 1 & $\mathbf{P}$ & C & $\mathbf{P}$ & C & $\mathbf{P}$ & C & $\mathbf{P}$ & C & $\mathbf{P}$ & $\mathrm{C}$ \\
\hline $\begin{array}{c}\text { Bare land } \\
(\mathrm{m} / \mathbf{s})\end{array}$ & \multicolumn{2}{|c|}{$3.99,-1.35$} & \multicolumn{2}{|c|}{$3.88,-1.35$} & \multicolumn{2}{|c|}{$3.99,-1.35$} & \multicolumn{2}{|c|}{$3.66,-1.35$} & \multicolumn{2}{|c|}{$3.38,-1.35$} \\
\hline $\begin{array}{c}\text { (Vegetation / } \\
\text { Bare land) }\end{array}$ & $\begin{array}{l}0.93, \\
0.59\end{array}$ & $\begin{array}{l}0.99 \\
0.66\end{array}$ & $\begin{array}{c}0.31 \\
0.79\end{array}$ & $\begin{array}{c}0.55 \\
0.87\end{array}$ & \begin{tabular}{|l|}
1.00, \\
1.44
\end{tabular} & $\begin{array}{l}1.00, \\
1.27\end{array}$ & $\begin{array}{l}1.00, \\
1.39\end{array}$ & $\begin{array}{l}1.00, \\
1.21\end{array}$ & $\begin{array}{l}1.04, \\
1.12\end{array}$ & $\begin{array}{l}1.01, \\
1.07\end{array}$ \\
\hline \multicolumn{11}{|l|}{ Case 2} \\
\hline $\begin{array}{l}\text { Bare land } \\
(\mathrm{m} / \mathrm{s})\end{array}$ & \multicolumn{2}{|c|}{$3.99,-1.35$} & \multicolumn{2}{|c|}{$3.88,-1.35$} & \multicolumn{2}{|c|}{$3.99,-1.35$} & \multicolumn{2}{|c|}{$3.66,-1.35$} & \multicolumn{2}{|c|}{$3.38,-1.35$} \\
\hline $\begin{array}{c}\text { (Vegetation / } \\
\text { Bare land) }\end{array}$ & $\begin{array}{c}0.93, \\
0.83\end{array}$ & $\begin{array}{l}0.99 \\
0.85\end{array}$ & $\begin{array}{c}0.51, \\
0.80\end{array}$ & $\begin{array}{c}0.71, \\
0.93\end{array}$ & \begin{tabular}{|l|}
1.00 \\
1.90
\end{tabular} & $\begin{array}{l}1.00, \\
1.53\end{array}$ & $\begin{array}{l}1.11, \\
1.65\end{array}$ & \begin{tabular}{|l|}
1.02, \\
1.39
\end{tabular} & $\begin{array}{l}1.28, \\
1.04\end{array}$ & $\begin{array}{l}1.17 \\
1.01\end{array}$ \\
\hline \multicolumn{11}{|l|}{ Case 3} \\
\hline $\begin{array}{c}\text { Bare land } \\
(\mathrm{m} / \mathrm{s})\end{array}$ & \multicolumn{2}{|c|}{$3.99,-1.35$} & \multicolumn{2}{|c|}{$2.92,-1.33$} & \multicolumn{2}{|c|}{$3.99,-1.35$} & \multicolumn{2}{|c|}{$3.44,-1.35$} & \multicolumn{2}{|c|}{$2.92,-1.33$} \\
\hline $\begin{array}{c}\text { (Vegetation / } \\
\text { Bare land) }\end{array}$ & $\begin{array}{l}0.93, \\
0.60 \\
\end{array}$ & $\begin{array}{l}0.99, \\
0.66\end{array}$ & $\begin{array}{c}0.46, \\
0.71 \\
\end{array}$ & $\begin{array}{c}0.68, \\
0.83 \\
\end{array}$ & \begin{tabular}{|l|}
1.00, \\
1.53 \\
\end{tabular} & $\begin{array}{l}1.00, \\
1.38\end{array}$ & $\begin{array}{l}1.02, \\
1.50\end{array}$ & $\begin{array}{l}1.01, \\
1.31 \\
\end{array}$ & $\begin{array}{l}0.97, \\
1.11 \\
\end{array}$ & $\begin{array}{l}0.96, \\
1.05\end{array}$ \\
\hline \multicolumn{11}{|l|}{ Case 4} \\
\hline $\begin{array}{c}\text { Bare land } \\
(\mathrm{m} / \mathrm{s})\end{array}$ & \multicolumn{2}{|c|}{$3.99,-1.35$} & \multicolumn{2}{|c|}{$2.92,-1.33$} & \multicolumn{2}{|c|}{$3.99,-1.35$} & \multicolumn{2}{|c|}{$3.44,-1.35$} & \multicolumn{2}{|c|}{$2.92,-1.33$} \\
\hline $\begin{array}{c}\text { (Vegetation / } \\
\text { Bare land) }\end{array}$ & $\begin{array}{l}0.93, \\
0.86\end{array}$ & $\begin{array}{l}0.99 \text {, } \\
0.90\end{array}$ & $\begin{array}{c}0.34, \\
0.75 \\
\end{array}$ & $\begin{array}{c}0.41, \\
0.89 \\
\end{array}$ & \begin{tabular}{|l|}
1.00, \\
2.05 \\
\end{tabular} & $\begin{array}{l}1.00, \\
1.67\end{array}$ & $\begin{array}{l}1.26, \\
1.80 \\
\end{array}$ & $\begin{array}{l}1.15, \\
1.49 \\
\end{array}$ & $\begin{array}{l}1.46, \\
0.97 \\
\end{array}$ & $\begin{array}{l}1.30, \\
0.98 \\
\end{array}$ \\
\hline
\end{tabular}


slope. It was observed when bed slope increased inundated maximum water depth increased while the maximum current velocity decreased. Change of the maximum water depth was insignificant for all patch arrangements. It was presumed that slope effect (or gravitational effect) was dominant than vegetation effect for maximum water depth variation. However vegetation patch effect remained for maximum current velocity variation for the relatively steep slope. It was observed that current velocity perpendicular to tsunami direction $(\mathrm{V})$ was less than $1.5 \mathrm{~m} / \mathrm{s}$ for all simulated cases. Contour lines were parallel (uniform slope) in tsunami direction. Hence this current velocity was generated due to resultant hydrostatic pressure between patch and gap. Table 8 shows the extreme horizontal run-up distances for different slopes with different patch arrangement. It was understood that

Table 8 Horizontal run-up calculated from shore line (m), B - Bare land, P - Pandanus and C - Casuarina

\begin{tabular}{|c|c|c|c|c|}
\hline & Case 1 & Case 2 & Case 3 & Case 4 \\
\hline 1:1000 - B & \multicolumn{5}{|c|}{2995} \\
\hline $\mathbf{1 : 1 0 0 0}-\mathbf{P}$ & 2875 & 2740 & 2850 & 2670 \\
\hline $\mathbf{1 : 1 0 0 0}-\mathbf{C}$ & 2905 & 2820 & 2880 & 2755 \\
\hline $\mathbf{1 : 1 0 0}-\mathbf{B}$ & \multicolumn{5}{|c|}{755} \\
\hline $\mathbf{1 : 1 0 0}-\mathbf{P}$ & 745 & 730 & 745 & 730 \\
\hline $\mathbf{1 : 1 0 0}-\mathbf{C}$ & 750 & 740 & 745 & 735 \\
\hline $\mathbf{1 : 5 0}-\mathbf{B}$ & \multicolumn{5}{|c|}{355} \\
\hline $\mathbf{1 : 5 0}-\mathbf{P}$ & 355 & 355 & 355 & 360 \\
\hline $\mathbf{1 : 5 0}-\mathbf{C}$ & 355 & 355 & 355 & 355 \\
\hline
\end{tabular}

when the slope become steep run-up distance reduced and vegetation effect was feeble. This was due to dominance of gravitational effect.

\section{CONCLUSION}

Two-dimensional numerical simulations were carried out to explore the vegetation patch effect on a tsunami event with different ground slope conditions. It was attempted to represent vegetation patch sizes available on the ground by changing their dimensions rationally. Additionally it was included gap between vegetation patches where it is common in real ground (gap can be represented in real case due to deforestation, access, etc). Simulation results can be summarized as fallows.

1) Out of selected vegetation species, Pandanus shows the high capability to resist a tsunami event than Casuarina. But difference is not so large. This was shown by $1-\mathrm{D}$ simulation too, Nandasena et al. ${ }^{2}$. Thus both vegetations would be introduced as a soft countermeasure for tsunami protection.
2) Vegetation patch can reduce maximum current velocity than maximum water depth behind the vegetation irrespective of ground slope. This was confirmed by one-dimensional numerical simulation with the assumption of infinitely long vegetation belt. Nandasena et al. ${ }^{2)}$.

3) When the ground slope becomes steep, reduction of maximum water depth behind the vegetation is reduced in comparison with bare land. This is due to dominant of gravity effect than vegetation effect.

4) Increment of patch size reduces the maximum water depth behind the vegetation on very mild slope, but for relatively steep slope, difference is negligible.

5) Horizontal run-up length is reduced with vegetation patch size increasing on very mild slope, but when the ground slope increases gravity effect beyond the vegetation effect on run-up length.

6) Relatively big vegetation patches with narrow gap can increase the maximum current velocity at the gap -outlet during tsunami flow landward and magnify at the gap-inlet when tsunami flow reversed in comparison with bare land. Maximum current velocity at the middle of the gap is always higher than the bare land case for both flow directions (landward / offshore). This indicates narrow gaps can be more destructive at a tsunami event.

\section{REFERENCES}

1) Nandasena, N. A. K., Tanaka, N., and Takagi, T.: A new derivation of horizontal two dimensional depth averaged momentum equation and continuity equation, which include total effect of porosity inside the vegetation, Annual Journal of Hydraulic Engineering, JSCE, VOL 52, pp.163-168, 2007.

2) Nandasena, N. A. K., Tanaka, N., and Tanimoto, K.: Capability of coastal vegetation species dominant in Asian region to retardate the tsunami impact, $9^{\text {th }}$ International Summer Symposium, JSCE, pp.115-118, 2007.

3) Tanaka, N., Sasaki, Y., Mowjood, M. I. M., Jinadasa, K. B. S. N.: Coastal vegetation structures and their functions in tsunami protection: Experience of the recent Indian Ocean tsunami, Landscape and ecological engineering, pp.33-45, 2007.

4) Tanaka, N., Nandasena, N. A. K., Jinadasa, K. S. B. N., Sasaki, Y., Tanimoto, K., and Mowjood, M. I. M.: Effective bio - shields structure for tsunami by coastal vegetation on sand dune, Civil Engineering and Environmental System, 2007, (Submitted).

5) Mascarenhas, A., and Jayakumar, S.: An environmental perspective of the post-tsunami scenario along the coast of Tamil Nadu, India: Role of sand dunes and forests, Journal of Environmental Management, 2007, (In press).

(Received September 30, 2007) 